\title{
Trends in Anemia Management in Hemodialysis Patients with Cancer
}

\author{
Anne M. Butler ${ }^{a, b} \quad$ Abhijit V. Kshirsagar ${ }^{b} \quad$ Andrew F. Olshan ${ }^{a, c}$ \\ Matthew E. Nielsen ${ }^{a, c-f}$ Stephanie B. Wheeler ${ }^{c, f}$ M. Alan Brookhart ${ }^{a, d}$ \\ ${ }^{a}$ Department of Epidemiology, ${ }^{b}$ UNC Kidney Center, ' Lineberger Comprehensive Cancer Center, ${ }^{d}$ Cecil G. Sheps \\ Center for Health Services Research, e Department of Urology, and fDepartment of Health Policy and Management, \\ University of North Carolina at Chapel Hill, Chapel Hill, N.C., USA
}

\section{Key Words}

Erythropoietin · Anemia - Iron · Blood transfusion · Dialysis · End-stage renal disease $\cdot$ Cancer

\begin{abstract}
Background: Erythropoiesis-stimulating agents (ESAs), intravenous iron, and blood transfusion are used to treat anemia in both end-stage renal disease (ESRD) and cancer. However, anemia treatment patterns have not been described among ESRD patients undergoing hemodialysis with concurrent cancer, especially in the recent era of ESA-related safety concerns. Methods: We analyzed Medicare data from a cohort of hemodialysis patients diagnosed with incident cancer. We used multivariable generalized linear models to estimate trends and patterns in ESA use, iron use, transfusion use, epoetin alfa (EPO) dose, iron dose, and resulting hemoglobin levels (2000-2011). Results: Of 43,760 eligible patients, quarterly ESA use declined slightly from a peak of 94.1 to $90.0 \%$. Quarterly EPO dose increased from 2000 to 2004, then declined; quarterly hemoglobin levels followed a similar pattern. Iron use increased rapidly from 46.9 to $79.3 \%$. Iron dose increased until 2010 and then declined. There was an increase in the quarterly transfusion use (6.3-11.7\%) and in the mean number of transfusion days per year (1.4-1.8). Anemia treatment patterns varied by demographic/clinical subgroups, especially among patients receiving chemotherapy, who required higher ESA use, EPO dose, and frequency
\end{abstract}

of transfusions. Conclusions: Despite safety concerns about ESAs in both the ESRD and cancer populations, the proportion of hemodialysis patients with cancer who used ESAs between 2000 and 2011 remained extremely high. EPO dose and hemoglobin levels increased and then decreased. Iron use, iron dose, and transfusions increased substantially. Future research examining the risk-benefit profile of different anemia management strategies in the dialysis population with cancer is needed.

(c) 2015 S. Karger AG, Basel

\section{Introduction}

Erythropoiesis-stimulating agents (ESAs) have been widely used for anemia management in both end-stage renal disease (ESRD) patients and cancer patients to increase hemoglobin levels, reduce the need for red blood cell transfusions, and alleviate anemia-related symptoms. After many years of escalating ESA use in both the ESRD population and the cancer population, anemia management patterns changed markedly in response to negative safety reports, product labeling changes, black box advisories, revised anemia management guidelines, and reimbursement changes.

In May 2004, the United States Food and Drug Administration (FDA) mandated the first of an increasingly restrictive series of product labeling changes for the ESA

\section{KARGER 125}

(C) 2015 S. Karger AG, Basel

0250-8095/15/0423-0206\$39.50/0
Anne M. Butler, $\mathrm{PhD}$

UNC Gillings School of Global Public Health

University of North Carolina at Chapel Hill

McGavran-Greenberg, CB \#7435, Chapel Hill, NC 27599-7435 (USA)

E-Mail anne.butler@unc.edu 
class of drugs in response to reports of increased mortality in patients with cancer [1]. In March 2007, the FDA mandated the addition of a black-box warning for ESA drugs due to reports of increased risk of death and serious cardiovascular events in patients with chronic kidney disease and increased risk of tumor progression and/or death in patients with cancer when hemoglobin levels were greater than $12.0 \mathrm{~g} / \mathrm{dl}$ [2]. This advisory was followed by revisions of clinical guidelines for ESA use [35]. Clinical guidelines have been regularly updated as emerging safety data have become available. Also in 2007, the Centers for Medicare and Medicaid Services published a National Coverage Decision to limit reimbursement for ESA administration and tie reimbursement to specific hemoglobin levels and time schedules [6]. Beginning in 2010, the FDA required that ESAs be prescribed to cancer patients under its risk evaluation and mitigation strategy program, which involves additional education for healthcare providers who prescribe and dispense ESAs as well as documentation that patients understand ESA-related risks [7]. In January 2011, CMS implemented the ESRD prospective payment system in which most aspects of the outpatient treatment of ESRD patients were combined into a single payment [8]. Shortly after, in June 2011, the FDA approved a revised ESA label that removed a specific target for hemoglobin level [9].

Several recent national studies conducted in both the cancer and chronic kidney disease populations have reported declines in ESA use, ESA dose, transfusion use, and hemoglobin levels and increases in iron use and iron dose [10-18]. However, no information exists regarding trends in anemia treatment among patients with both ESRD and cancer.

A cancer diagnosis complicates anemia management in ESRD patients undergoing dialysis for many reasons. First, there are additional safety concerns associated with ESA treatment in the cancer population including tumor progression, thromboembolic complications, and mortality $[19,20]$. Beginning in 2003, 8 randomized clinical trials in cancer patients reported increased risk of tumor progression and/or death among cancer patients treated with ESAs [21-29]. The most recent meta-analyses of cancer patients demonstrated significant effects of ESAs on increased thromboembolic events and mortality but not on disease progression [30]. Second, there are currently no formal guidelines for providers regarding appropriate usage of ESAs in ESRD patients with cancer [20]. For patients undergoing dialysis, the United States recommendation is to initiate treatment when the hemoglobin level is less than $10 \mathrm{~g} / \mathrm{dl}$ and to individualize dos- ing, and use the lowest dose of ESA sufficient to reduce the need for transfusions [31]. For cancer patients, the recommendations are similar except that the ESA indication for treatment of anemia is restricted to patients undergoing myelosuppressive chemotherapy [32]. Third, little is known regarding patterns of anemia management in dialysis patients with cancer. In this population, it remains unclear how anemia treatment has been affected by recent clinical and policy events.

The objectives of this study were to examine trends in anemia management in the United States hemodialysis population after the diagnosis of cancer. Using population-based data from the United States Renal Data System (USRDS), which is a national registry including all patients in Medicare's ESRD program, we described patterns of use of ESAs, iron, and transfusions as well as resulting hemoglobin levels. We examined anemia management patterns within subgroups, including cancer site and chemotherapy use. We report trends from 2000 to 2011, a time period that includes data before and after negative safety reports, product labeling changes, black box advisories, revised anemia management guidelines, and reimbursement changes.

\section{Methods}

\section{Study Population}

Using data from the USRDS, a national registry of patients in Medicare's ESRD program, we identified all ESRD patients $\geq 18$ years who received in-center hemodialysis between April 1, 1995 and December 31, 2011, with Medicare as their primary payer and with both Parts A and B coverage. We restricted the cohort to patients who received their first cancer diagnosis at least 9 months after dialysis initiation. This time period was to ensure stability in dialysis treatment modality (months $0-3$ post-dialysis initiation) [33], and to exclude prevalent cancer cases identified using Medicare claims (months 3-8 post-dialysis initiation), as previously described [34-37]. Patients with a malignancy-related primary cause of ESRD or a history of kidney transplantation or HIV/AIDS were also excluded. We further restricted the cohort to patients with post-cancer diagnosis follow-up time between 2000 and 2011. This restriction ensured a similar distribution of incident and prevalent hemodialysis patients over time, which was necessary because epoetin alfa (EPO) dosing has been documented to increase with longer time on dialysis [15].

\section{Incident Cancer Definitions}

We identified incident site-specific cancers using International Classification of Diseases, 9th revision, Clinical Modification (ICD-9-CM) diagnosis codes from inpatient and outpatient Medicare claims (online suppl. table 1; for all online suppl. material, see www.karger.com/doi/10.1159/000440771), as previously described [34]. The claims-based algorithm used to define site-specific cancers required $\geq 2$ ICD-9-CM diagnosis codes within 
6 months, adapted from an algorithm (requiring $\geq 2$ ICD-9-CM diagnosis codes within 2 months) validated in a Medicare population for identification of incident cancers including lung, colorectal, stomach, breast and lymphoma (sensitivity: range 56-77\%; specificity: $\geq 99 \%$; positive predictive value: range $56-77 \%$ ) [38]. We extended the time period to 6 months between cancer diagnosis codes to allow for delays in health care encounters that may occur due to the high severity of illness characteristic of the ESRD population. Date of cancer onset was defined as the first date of a cancer-related diagnosis code in the claims data. In situ carcinomas were included for 2 sites (i.e. breast and bladder) [39]. Secondary tumors, benign tumors, and non-melanoma skin cancers were excluded. Only the first cancer diagnosis after dialysis initiation was included as an event.

\section{Anemia Therapy Outcomes}

Information on ESAs, iron, and hematocrit was identified from dialysis claims from the Medicare Part A institutional claims files and Medicare Parts A and B claims. Information on transfusions was identified from Medicare Parts A and B claims. The codes used to identify ESAs, iron, and transfusions are presented in online supplemental table 2 . Monthly outlier values were set to missing if they were outside the specified ranges (EPO dose 500-700,000 units; hematocrit 20-60 g/dl) [15]. Data were summarized by calendar quarter. We calculated the quarterly proportion of the study population treated with ESAs (EPO, darbepoetin alfa (DPO)) or iron. Mean EPO dose (units/month) was calculated as the quarterly sum of EPO doses divided by 3. DPO dose was excluded from the analysis due to major variation in annual missingness over the study period. Because most DPO use occurred in hospital-based facilities, these units were excluded from the EPO dose calculation to avoid under-ascertainment of total ESA dose. Iron dose (mg) was based on the Healthcare Common Procedure Coding System code for iron dextran, iron sucrose, ferric gluconate, or ferumoxytol, and number of units associated with each claim. Mean quarterly hemoglobin levels were calculated by dividing hematocrit levels by 3 . We calculated the quarterly proportion of the study population that received transfusions, as well as the mean number of days per year that each patient received a transfusion.

\section{Covariates}

We obtained the following information from the USRDS patient file: first service date, sex, race, and primary cause of ESRD. Information on ethnicity was obtained from the Medical Evidence file. For each calendar quarter, age at dialysis initiation and duration of dialysis (i.e. vintage) were calculated on the first date of the quarter by subtracting the first service date. We identified chemotherapy administration during each quarter using ICD-9-CM diagnosis and procedure codes, Healthcare Common Procedure Coding System codes, and revenue codes (online suppl. table 3) $[40,41]$.

\section{Statistical Analysis}

We calculated descriptive statistics on the demographic and clinical characteristics of the population by year. We also described the annual distribution of cancer diagnoses by cancer site. To describe trends in anemia treatment by calendar quarter, we used generalized linear models to generate unadjusted and adjusted estimates. Specifically, we used logistic regression models to calculate quarterly estimates of the proportion of patients that used
ESAs, iron, or transfusion, linear regression models to calculate mean quarterly estimates of EPO dose (units/month), iron dose (mg/quarter) and hemoglobin levels ( $\mathrm{g} / \mathrm{dl}$ ), and Poisson regression models to calculate estimates of the mean number of transfusion days per year. Since EPO dose and hematocrit levels were reported only with the administration of ESAs, these analyses were restricted to patients who received ESAs at least once during the quarter. Calendar quarter or year was treated as a categorical variable in the models to relax the assumption of linearity. The results section presents plots of the adjusted estimates for ESA use, iron use, transfusion use, EPO dose, iron dose, number of transfusion days, and hemoglobin levels, which are population marginal means that account for changes over time in demographic (age, sex, race, ethnicity) and clinical characteristics (primary cause of ESRD, dialysis vintage) [42]. The trend lines and corresponding 95\% CI were calculated as a smoothed conditional mean of the quarterly or yearly adjusted estimates. We stratified estimates by cancer site and chemotherapy use, as well as several additional demographic and clinical characteristics. We further stratified the analyses by using 3 categories of cancer site: (a) all cancer sites; (b) the most common solid tumors in our study population (i.e. cancers of the prostate, female breast, colon/rectum, lung/bronchus, kidney/renal pelvis, bladder, and pancreas); and (c) hematologic malignancies (i.e. Hodgkin lymphoma, non-Hodgkin lymphoma (NHL), leukemia, and myeloma). We performed complete case analyses since covariate missingness was less than $1 \%$ (i.e. ethnicity $(\mathrm{n}=156))$. SAS software version 9.3 (Cary, N.C., USA) and R, version 0.98.507 were used to perform analyses [43].

\section{Results}

A total of 43,760 incident cancer patients receiving hemodialysis met eligibility requirements for this study. Demographic and clinical characteristics are presented for selected years (i.e. 2000, 2002, 2004, 2007, 2009, and 2011 ) in table 1 . The number of eligible patients per year increased from 5,081 in 2000 to 11,898 in 2011. Over time, patients were more likely to be black, Hispanic, or have diabetes as the primary cause of ESRD. Between 2000 and 2011, mean age at dialysis initiation decreased from 67.9 to 65.7 years, whereas the mean age at cancer diagnosis remained constant. The median duration of dialysis at cancer diagnosis was 2.1 years (IQR 1.3-3.6). The median length of follow-up after cancer diagnosis was 1.0 year (IQR $0.3-2.4$ ), and $19.2 \%$ of patients remained in follow-up at 3 years post-cancer diagnosis.

The distribution of cancer diagnoses by cancer site and year is presented in online supplemental figure 1 and table 4 . The most frequently diagnosed cancer sites were prostate, colorectal, and the female breast. Cancers of the kidney/renal pelvis had the most notable increase between 2000 and 2011, from 6.5 to $11.2 \%$ of diagnosed cancers. 
Table 1. Demographic and clinical characteristics of the hemodialysis population with cancer in 2000, 2002, 2004, 2007, 2009, and 2011

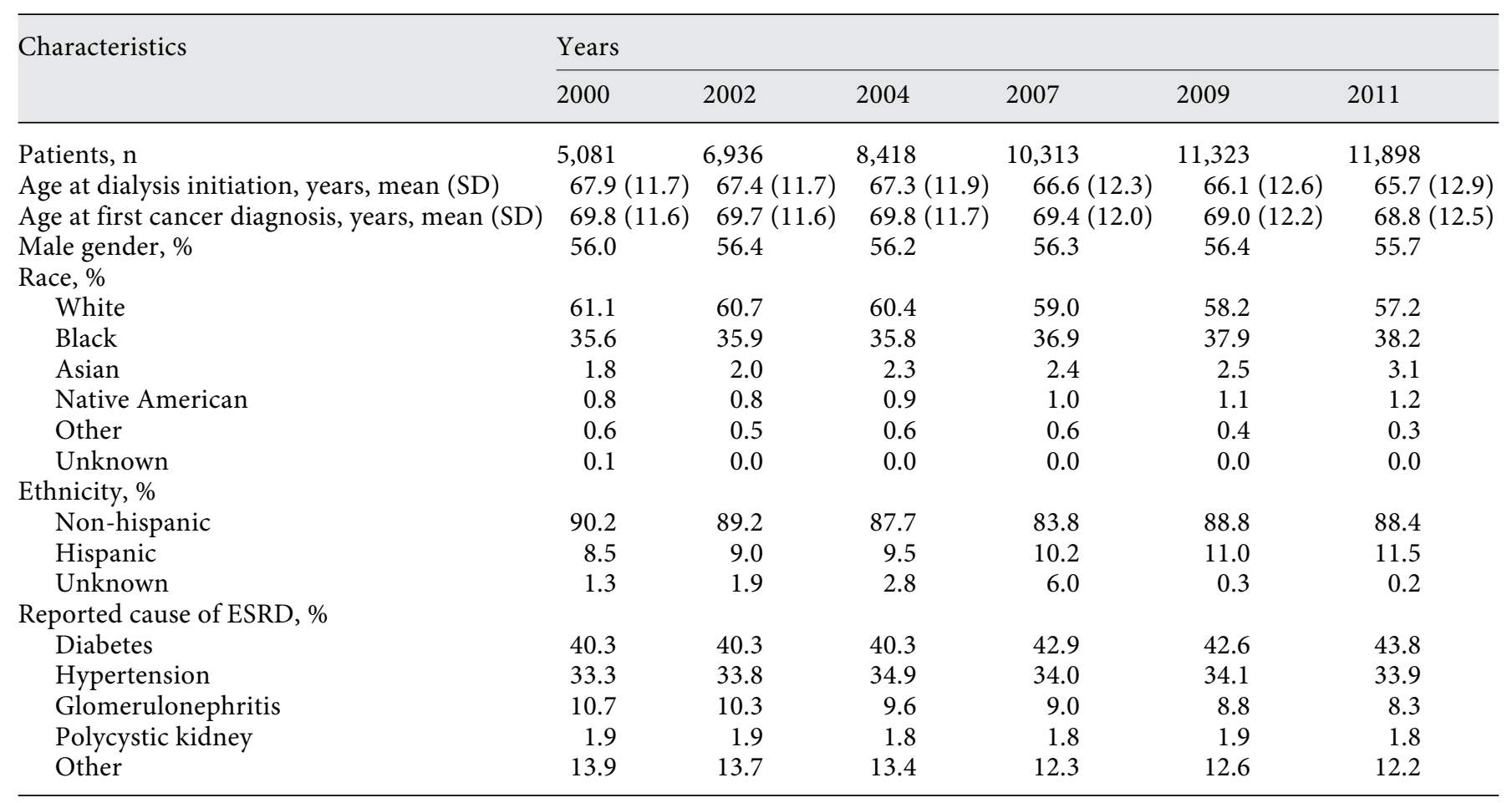

* Patients may be included in multiple years.

Over the study period, the quarterly proportion of patients that received ESAs declined slightly, from a peak of $94.1 \%$ in early 2002 to $90.0 \%$ in late 2011 . Use of EPO decreased from 94.1 to $85.4 \%$ between mid-2002 and mid-2007, and then remained relatively stable. Use of DPO increased from 0.0 to $7.9 \%$ between 2003 and 2007, and then decreased to $5.2 \%$ by 2011 (online suppl. fig. 2). ESA use varied by cancer site. On average, quarterly ESA use was lower among patients with hematologic malignancies compared to patients with solid tumors ( $92.0 \mathrm{vs.}$ 93.3\%; fig. 1; online suppl. fig. 3, 4). The quarterly proportion of patients that received intravenous iron increased gradually from $46.9 \%$ in late 2001 to $79.3 \%$ in mid-2011, and then declined to $73.9 \%$ by late 2011 . On average, quarterly iron use was lower among patients with hematologic malignancies compared to patients with solid tumors (62.4 vs. $66.5 \%$; fig. 1; online suppl. fig. 3-5).

Among patients receiving EPO, the mean EPO dose increased from approximately 66,000 units/month in early 2001 to 83,000 units/month in mid-2004, and then declined to 52,000 units/month by late 2011 . Patients with hematologic malignancies used a higher mean EPO dose compared to patients with solid tumors $(\sim 86,000$ vs. 72,000 units/month; fig. 2; online suppl. fig. 3, 4).

Among patients receiving iron, the mean dose increased from approximately $520 \mathrm{mg}$ in 2000 to 830 in 2010 , and then declined to $680 \mathrm{mg}$ in 2011. Iron dose did not vary substantially by cancer site (fig. 2; online suppl. fig. 3, 4).

Mean quarterly hemoglobin levels followed a similar pattern to EPO dose. Among patients receiving ESAs, hemoglobin levels increased from $11.3 \mathrm{~g} / \mathrm{dl}$ in early 2000 to $11.9 \mathrm{~g} / \mathrm{dl}$ in mid-2004, and then declined to $10.7 \mathrm{~g} / \mathrm{dl}$ by late 2011. Patients with hematologic malignancies had slightly lower mean quarterly hemoglobin levels compared to patients with the most common solid tumors (11.4 vs. 11.6 g/dl; fig. 2; online suppl. fig. 3,4 ).

The quarterly proportion of patients that received transfusions increased from $6.3 \%$ in mid-2000 to $11.7 \%$ in mid-2011. Use of transfusion was more common among patients with hematologic malignancies compared to patients with solid tumors (quarterly mean, 12.0 vs. $8.2 \%$; fig. 3; online suppl. fig. 3, 4). Among patients who received transfusion, the mean number of transfusion days per year increased steadily from 1.5 to 1.9 days 
Fig. 1. Mean quarterly ESA use and iron use for all cancer sites; most common solid tumors in our study population (i.e. cancers of the prostate, female breast, colon/ rectum, lung/bronchus, kidney/renal pelvis, bladder, and pancreas); and hematologic malignancies (i.e. Hodgkin lymphoma, NHL, leukemia, and myeloma). Quarterly data points were adjusted for age, sex, race, ethnicity, cause of ESRD, and dialysis vintage. Trend lines represent smoothed conditional means.

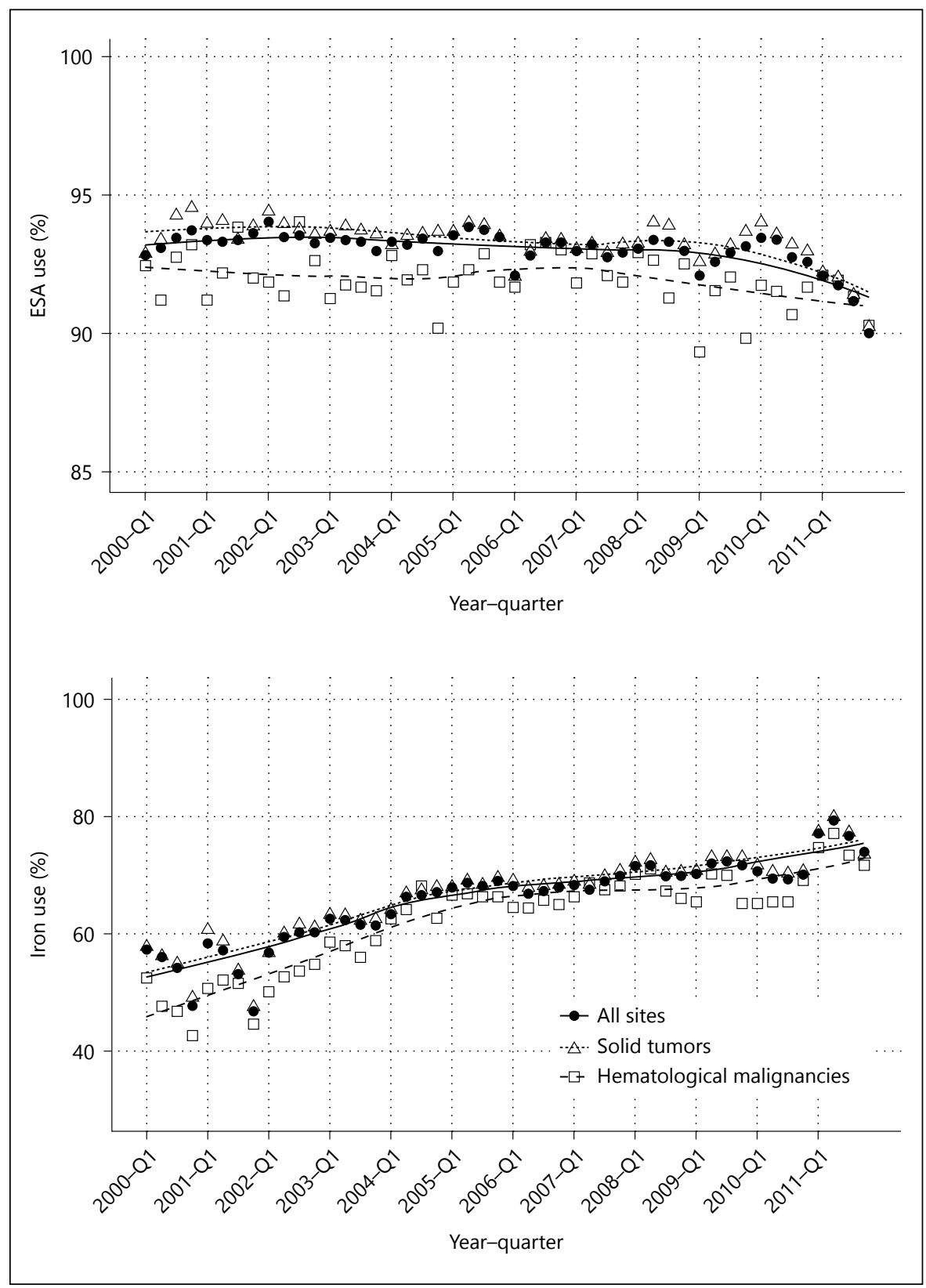

between 2000 and 2011, with a steeper increase among patients with hematologic malignancies (1.6-2.6 days) compared to patients with solid tumors (1.5-1.8 days; fig. 3).

The quarterly proportion of chemotherapy receipt decreased from $6.3 \%$ in quarter 1 of 2000 to $5.8 \%$ in quarter 4 of 2011. Online supplemental figure 6 presents quarterly trends in anemia treatment by chemotherapy use. Compared to cancer patients who did not receive chemotherapy, patients who received chemotherapy had higher mean quarterly ESA use (93 vs. $96 \%$ ) and higher mean EPO dose ( 72,000 vs. 95,000 units/month). Almost twice as many patients on chemotherapy received quarterly transfusions compared to patients who did not receive chemotherapy (15.8 vs. $8.3 \%$ ). Mean quarterly hemoglobin levels were lower among patients who received chemotherapy compared to patients who did not receive chemotherapy (11.3 vs. $11.6 \mathrm{~g} / \mathrm{dl}$ ).

Analyses stratified by demographic characteristics revealed several meaningful differences in anemia treat- 
Fig. 2. Mean quarterly ESA dose, iron dose, and hemoglobin levels for all cancer sites; most common solid tumors in our study population (i.e. cancers of the prostate, female breast, colon/rectum, lung/bronchus, kidney/renal pelvis, bladder, and pancreas); and hematologic malignancies (i.e. Hodgkin lymphoma, NHL, leukemia, and myeloma). Quarterly data points were adjusted for age, sex, race, ethnicity, cause of ESRD, and dialysis vintage. Trend lines represent smoothed conditional means. For EPO dose, patients treated at a hospital-based facility were excluded to remove the possibility of simultaneous treatment with EPO and DPO.

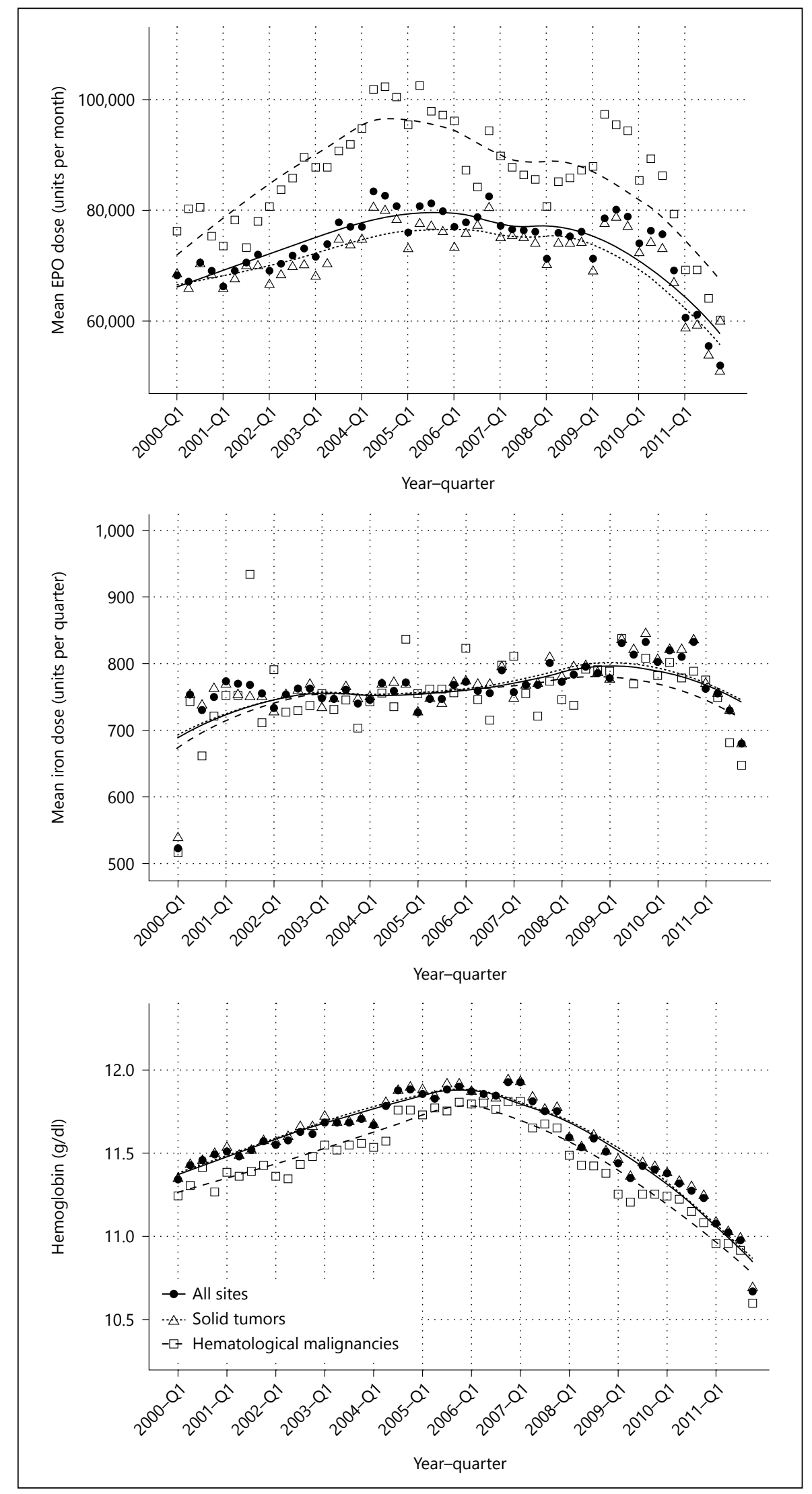


Fig. 3. Mean quarterly use of blood transfusions and mean number of blood transfusion days per year among patients who received blood transfusions, by cancer site for all cancer sites; most common solid tumors in our study population (i.e. cancers of the prostate, female breast, colon/rectum, lung/bronchus, kidney/renal pelvis, bladder, and pancreas); and hematologic malignancies (i.e. Hodgkin lymphoma, NHL, leukemia, and myeloma). Data points were adjusted for age, sex, race, ethnicity, cause of ESRD, and dialysis vintage. Trend lines represent smoothed conditional means.

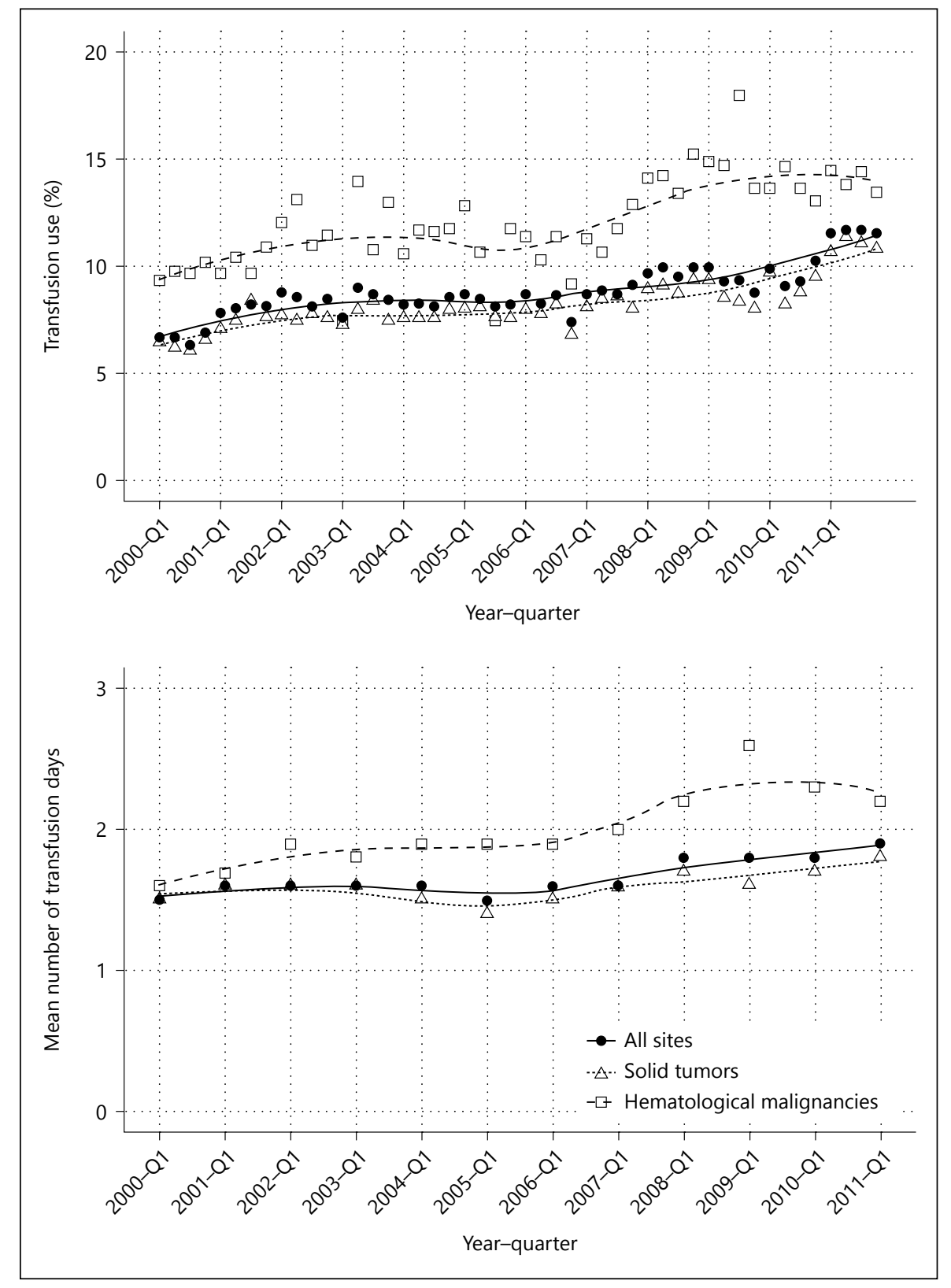

ment patterns (online suppl. fig. 7-12). ESA use was lower among patients who were male, non-African American, receiving dialysis for $<2$ years, or with primary cause of ESRD due to polycystic kidney disease. EPO dose was lower among patients who were older, non-African American, Hispanic, receiving dialysis for $<2$ years, or with primary cause of ESRD due to polycystic kidney disease. Iron use was lower among patients who were older or who belonged to another race. Iron dose was lower among patients who were older, female, non-African American, or receiving dialysis for $<2$ years. Transfusion use was lower among patients who were non-Hispanic, receiving dialysis for $\geq 2$ years, or with primary cause of ESRD due to polycystic kidney disease. Hemoglobin levels did not vary substantially by subgroups.

\section{Discussion}

In this study of anemia treatment patterns among United States hemodialysis patients diagnosed with incident cancer, we observed high and near-constant use of 
ESAs. Compared to previously reported trends in the population of hemodialysis patients without cancer, ESA use remained higher and more constant (i.e. $>90 \%$ ), but EPO dosing and hemoglobin level estimates were similar (i.e. increased until 2004 and 2006, respectively, and then declined steadily). We observed significant variability in ESA use and EPO dosing across subgroups. Specifically, ESA use was lower among patients who were male, nonAfrican American, had been receiving dialysis for less than 2 years, or had polycystic kidney disease as cause of ESRD. And notably, average EPO doses were substantially higher among patients with hematological malignancies as well as patients receiving chemotherapy compared to patients with solid tumors and no chemotherapy, respectively. Additionally, we noted a similarly steady increase in iron use, iron dose, and transfusion use.

To our knowledge, this is the first study in the hemodialysis population with incident cancer examining trends in anemia management. In contrast to our findings of constant ESA use over time in our hemodialysis population with cancer, the cancer literature documents a dramatic decrease in ESA use (i.e. both EPO and DPO) among cancer patients in the years surrounding the 2007 FDA black-box warning. Among VA cancer patients, ESA use plateaued in late 2003 and sharply declined from 38\% (2006) to $12 \%$ (2008) among colon cancer patients and from $23 \%$ (2006) to 5\% (2008) among lung cancer patients [10]. Another study of cancer patients undergoing chemotherapy across 39 sites in 7 states reported a decline from 41 to $30 \%$ between 10-month intervals before and after the 2007 FDA black-box warning [12]. Two studies of cancer patients treated at MD Anderson Comprehensive Cancer Center reported decreases in ESA use between 2006 and 2008 of 17 to 5 and 4 to $1 \%$, respectively $[11,13]$.

Although ESA use remained relatively constant among dialysis patients with cancer over the study period, we observed an 8.7\% decrease in EPO use between 2002 and 2007 and a corresponding increase in DPO use. Indeed, the timing and magnitude of the decline in EPO use follow a pattern similar to that previously documented in the hemodialysis population [15]. In both studies, EPO use declined steeply after the emergence of ESA-related safety concerns and has continued to decline. Compared to the larger hemodialysis population, our hemodialysis population with cancer had slightly lower EPO use and hemoglobin levels, slightly higher EPO dosing, and similar iron use and dosing. Among hemodialysis patients with cancer, ESA use remains high. However, the administered dose has declined substantially for a majority of these patients.

Anemia Management in ESRD, 2000-2010
After the 2011 CMS implementation of the new bundled prospective payment system, we observed a decrease in ESA use, EPO dose, iron dose, and hemoglobin levels and an increase in iron use and transfusion use [8]. These results were consistent with reports from other populations [17, 44]. It is important to continue to monitor these trends.

There is a conflict in the current guidelines regarding appropriate treatment in the subgroup of dialysis patients with cancer who do not receive chemotherapy. The guidelines for dialysis patients are similar to the guidelines for cancer patients, except that the ESA indication for treatment of anemia is restricted to cancer patients undergoing myelosuppressive chemotherapy [31,32]. These evidencebased guidelines were influenced by studies of cancer patients that consistently reported modification of mortality risk associated with ESAs by chemotherapy use [20]. As expected, we observed that patients who did not receive chemotherapy had higher hemoglobin levels and less intensive anemia treatment, including lower ESA use, EPO dose, and transfusion use, compared to patients that received chemotherapy. Yet, the common ESA use in this subgroup of patients not receiving myelosuppressive chemotherapy is noteworthy. These findings highlight discrepancies between guideline-recommended therapy and actual practice.

Transfusion avoidance remains an important goal due to dangerous transfusion-related complications including hyperkalemia, fluid overload, iron overload and allosensitization [45]. Between 2000 and 2011, we observed an increasing proportion of hemodialysis patients with cancer that received transfusions and an increasing mean number of transfusion days per year. Since transfusion avoidance provided the formal reason for the original approval and use of ESAs, it seems paradoxical that transfusion use is increasing despite constant ESA use [46]. One likely explanation for the increase in transfusion use is the recent paradigm shift toward lower ESA dosing and lower hemoglobin levels. A recent study reported that Medicare hemodialysis patients with 3-month mean hemoglobin levels $<10 \mathrm{~g} / \mathrm{dl}$ received transfusions at a rate approximately 4 times higher than the rate for patients with hemoglobin levels maintained at $\geq 10 \mathrm{~g} / \mathrm{dl}$. Although transfusion rates among patients with hemoglobin levels $<10$ and $\geq 10 \mathrm{~g} / \mathrm{dl}$ remained relatively constant between 1999 and 2010, the proportion of patients with hemoglobin levels $<10 \mathrm{~g} / \mathrm{dl}$ began to increase after 2006, and consequently, the absolute number of patients receiving transfusions also began to increase [16].

Our findings are subject to several limitations. First, our results may not be generalizable to non-dialysis pa-

Am J Nephrol 2015;42:206-215 
tients with chronic kidney disease, patients on peritoneal dialysis, patients with a non-Medicare primary payer, or patients who died within 9 months of dialysis initiation. Second, claims-based cancer definitions are commonly used in cancer research, but have not been validated in the ESRD population. Third, we cannot exclude the possibility of misclassifying prevalent cancer cases as incident cases. However, identification of prevalent cancer cases during the 6-month baseline period minimized the possibility of misclassification. Fourth, the use of claimsbased definitions made it impossible to determine whether cancers identified as incident cases were truly new primaries, metastases, or histories of cancer that were miscoded as new primaries. Fifth, we analyzed the number of transfusion days per year rather than the actual number of transfusions, because all transfusions administered on any single day are covered by a single procedural code. Sixth, ESA claims are provided from outpatient dialysis claims but not from hospital claims. Since ESRD patients require several hospital days per year on average, this missing data may yield lower estimates of ESA use and dose. Lastly, we may be missing a small proportion of chemotherapy use due to lack of access to some specific chemotherapy agent codes (i.e. National Drug Codes).

Strengths of our study include more than a decade of data on the large and representative population of United States ESRD patients diagnosed with cancer after hemodialysis initiation. The large sample size allowed the novel characterization of anemia management patterns within subgroups, including cancer site and chemotherapy use.
In conclusion, our results suggest that ESA and iron use is common in hemodialysis patients with cancer, and trends in dosing have been similar to those in the overall hemodialysis population. The potential risks associated with ESA and iron use must be balanced with known benefits, such as alleviating anemia-related symptoms and transfusion avoidance. Further research is needed to better understand the risks and benefits of anemia therapy in the population of dialysis patients with cancer.

\section{Acknowledgments}

A.M.B. was supported by the NIH NIDDK (2 T32 DK00775015, PI: R.J. Falk) and NCI (5 T32 CA009330-30, PI: A.F. Olshan). M.A.B. receives investigator-initiated research funding from the National Institutes of Health (R01 AG042845, R21 HD080214, R01 AG023178) and through contracts with the Agency for Healthcare Research and Quality's DEcIDE program and the Patient Centered Outcomes Research Institute.

Some of the data reported here have been supplied by the USRDS. The interpretation and reporting of these data are the responsibility of the authors and in no way should be seen as an official policy or interpretation of the United States government.

\section{Disclosure Statement}

M.A.B. reports receiving grant support from Amgen; serving on scientific advisory boards for Pfizer, Amgen, and Merck (honoraria declined, donated, or received by institution); and serving as a consultant to RxAnte and World Health Information Consultants. A.V.K. reports serving on an advisory board for Fresenius. No other authors report competing financial interests.

\section{References}

1 United States Food and Drug Administration: FDA public health advisory: erythropoiesisstimulating agents (ESAs). http://www.access data.fda.gov/drugsatfda_docs/nda/2004/ 103234s5033.pdf (accessed April 9, 2014).

2 United States Food and Drug Administration: FDA public health advisory: erythropoiesisstimulating agents (ESAs). 2007. http://www. accessdata.fda.gov/drugsatfda_docs/label/ 2007/103234s5122lbl.pdf (accessed April 9, 2013).

3 Rizzo JD, Somerfield MR, Hagerty KL, et al: Use of epoetin and darbepoetin in patients with cancer: 2007 American Society of Clinical Oncology/American Society of Hematology clinical practice guideline update. J Clin Oncol 2008;26:132-149.

4 KDOQI clinical practice guideline and clinical practice recommendations for anemia in chronic kidney disease: 2007 update of hemo- globin target. Am J Kidney Dis 2007;50:471530.

5 Rodgers GM 3rd, Becker PS, Bennett CL, et al: Cancer- and chemotherapy-induced anemia. J Natl Compr Canc Netw 2008;6:536-564.

6 Phurrough S, Jacques L, Ciccanti M, Long K, Koller E: Decision Memo for Erythropoiesis Stimulating Agents (ESAs) for Non-Renal Disease Indications (CAG-00383N). US Department of Health and Human Services Centers for Medicare and Medicaid Services, 2007.

7 US Department of Health and Human Services; US Food and Drug Administration: Approved risk evaluation and mitigation strategies (REMS). http://www.fda.gov/Drugs/Drug Safety/PostmarketDrugSafetyInformationfor PatientsandProviders/ucm111350.htm (accessed July 24, 2014)

8 Centers for Medicare \& Medicaid Services (CMS), HHS: Medicare program; end-stage renal disease prospective payment system. Final rule. Fed Regist 2010;75:49029-49214.

9 US Food and Drug Administration; US Department of Health and Human Services: FDA Drug Safety Communication: Modified Dosing Recommendations to Improve the Safe Use of Erythropoiesis-Stimulating Agents (ESAs) in Chronic Kidney Disease. Safety Announcement, 2011.

10 Tarlov E, Stroupe KT, Lee TA, et al: Trends in anemia management in lung and colon cancer patients in the US Department of Veterans Affairs, 2002-2008. Support Care Cancer 2012;20:1649-1657.

11 Ferrajoli A, Buzdar AU, Dejesus Y, Cheng L, Michaud LB, Rodriguez MA: Usage of erythropoiesis-stimulating agents in cancer patients at an academic cancer center and experience with specific care management tools for anemia. Cancer 2011;117:3268-3275. 
12 Hess G, Nordyke RJ, Hill J, Hulnick S: Effect of reimbursement changes on erythropoiesisstimulating agent utilization and transfusions. Am J Hematol 2010;85:838-843.

13 Vadhan-Raj S, Zhou X, Sizer K, et al: Impact of safety concerns and regulatory changes on the usage of erythropoiesis-stimulating agents and RBC transfusions. Oncologist 2010;15: 1359-1369.

14 Lal LS, Raju A, Miller LA, Chen H, Arbuckle R, Sansgiry SS: Impact of changes in reimbursement policies and institutional practice algorithm for utilization of erythropoieticstimulating agents on treatment patterns and costs in anemic lymphoma patients. Support Care Cancer 2010;19:251-259.

15 Freburger JK, Ng LJ, Bradbury BD, Kshirsagar $\mathrm{AV}$, Brookhart MA: Changing patterns of anemia management in US hemodialysis patients. Am J Med 2012;125:906-914.e9.

16 Gilbertson DT, Monda KL, Bradbury BD, Collins AJ: RBC transfusions among hemodialysis patients (1999-2010): influence of hemoglobin concentrations below $10 \mathrm{~g} / \mathrm{dl}$. Am J Kidney Dis 2013;62:919-928.

17 Fuller DS, Pisoni RL, Bieber BA, Port FK, Robinson BM: The DOPPS practice monitor for U.S. dialysis care: update on trends in anemia management 2 years into the bundle. Am J Kidney Dis 2013;62:1213-1216.

18 Winkelmayer WC, Mitani AA, Goldstein BA, Brookhart MA, Chertow GM: Trends in anemia care in older patients approaching endstage renal disease in the United States (19952010). JAMA Intern Med 2014;174:699-707.

19 Kidney Disease: Improving Global Outcomes (KDIGO) Anemia Work Group. KDIGO Clinical Practice Guideline for Anemia in Chronic Kidney Disease. Kidney Int Suppl 2012;2:279335.

20 Hazzan AD, Shah HH, Hong S, Sakhiya V, Wanchoo R, Fishbane S: Treatment with erythropoiesis-stimulating agents in chronic kidney disease patients with cancer. Kidney Int 2014;86:34-39.

21 Leyland-Jones B; BEST Investigators and Study Group: Breast cancer trial with erythropoietin terminated unexpectedly. Lancet Oncol 2003;4:459-460.

22 Leyland-Jones B, Semiglazov V, Pawlicki M, et al: Maintaining normal hemoglobin levels with epoetin alfa in mainly nonanemic patients with metastatic breast cancer receiving first-line chemotherapy: a survival study. J Clin Oncol 2005;23:5960-5972.

23 Henke M, Laszig R, Rübe C, et al: Erythropoietin to treat head and neck cancer patients with anaemia undergoing radiotherapy: randomised, double-blind, placebo-controlled trial. Lancet 2003;362:1255-1260.
24 Hedenus M, Adriansson M, San Miguel J, et al: Efficacy and safety of darbepoetin alfa in anaemic patients with lymphoproliferative malignancies: a randomized, double-blind, placebo-controlled study. Br J Haematol 2003;122: 394-403.

25 Wright JR, Ung YC, Julian JA, et al: Randomized, double-blind, placebo-controlled trial of erythropoietin in non-small-cell lung cancer with disease-related anemia. J Clin Oncol 2007;25:1027-1032.

26 Smith RE Jr, Aapro MS, Ludwig H, et al: Darbepoetin alpha for the treatment of anemia in patients with active cancer not receiving chemotherapy or radiotherapy: results of a phase III, multicenter, randomized, double-blind, placebo-controlled study. J Clin Oncol 2008; 26:1040-1050.

27 Untch M, von Minckwitz G, Konecny GE, et al: PREPARE trial: a randomized phase III trial comparing preoperative, dose-dense, dose-intensified chemotherapy with epirubicin, paclitaxel, and CMF versus a standarddosed epirubicin-cyclophosphamide followed by paclitaxel with or without darbepoetin alfa in primary breast cancer - outcome on prognosis. Ann Oncol 2011;22:1999-2006.

28 Thomas G, Ali S, Hoebers FJ, et al: Phase III trial to evaluate the efficacy of maintaining hemoglobin levels above $12.0 \mathrm{~g} / \mathrm{dl}$ with erythropoietin vs above $10.0 \mathrm{~g} / \mathrm{dl}$ without erythropoietin in anemic patients receiving concurrent radiation and cisplatin for cervical cancer. Gynecol Oncol 2008;108:317-325.

29 Overgaard J, Hoff C, San Hansen H, et al: Randomized study of the importance of novel erythropoiesis stimulating protein (Aranesp ${ }^{\circledR}$ ) for the effect of radiotherapy in patients with primary squamous cell carcinoma of the head and neck (HNSCC) - the Danish Head and Neck Cancer Group DAHANCA 10 randomized trial. Eur J Cancer 2007;5:6LB.

30 Tonia T, Mettler A, Robert N, et al: Erythropoietin OR darbepoetin for patients with cancer. Cochrane Database Syst Rev 2012;12: CD003407.

31 FDA Drug Safety Communication: Modified dosing recommendations to improve the safe use of erythropoiesis-stimulating agents (ESAs) in chronic kidney disease.

32 FDA Announces New Safety Plan for Agents Used to Treat Chemotherapy-Related Anemia. Administration UFaD, trans.

33 System URD: Researcher's Guide to the USRDS Database: 2013 ADR Edition. Bethesda, National Institutes of Health, National Institute of Diabetes and Digestive and Kidney Diseases, 2013.
34 Butler AM, Olshan AF, Kshirsagar AV, et al: Cancer incidence among US Medicare ESRD patients receiving hemodialysis, 1996-2009. Am J Kidney Dis 2015;65:763-772.

35 Kshirsagar AV, Freburger JK, Ellis AR, Wang L, Winkelmayer WC, Brookhart MA: The comparative short-term effectiveness of iron dosing and formulations in US hemodialysis patients. Am J Med 2013;126:541.e1e14.

36 Brookhart MA, Freburger JK, Ellis AR, Wang L, Winkelmayer WC, Kshirsagar AV: Infection risk with bolus versus maintenance iron supplementation in hemodialysis patients. J Am Soc Nephrol 2013;24:1151-1158.

37 Beaubrun AC, Kilpatrick RD, Freburger JK, Bradbury BD, Wang L, Brookhart MA: Temporal trends in fracture rates and postdischarge outcomes among hemodialysis patients. J Am Soc Nephrol 2013;24:14611469.

38 Setoguchi S, Solomon DH, Glynn RJ, Cook EF, Levin R, Schneeweiss S: Agreement of diagnosis and its date for hematologic malignancies and solid tumors between Medicare claims and cancer registry data. Cancer Causes Control 2007; 18:561-569.

39 Howlader N, Noone A, Krapcho M, et al: SEER Cancer Statistics Review, 1975-2010. Bethesda, National Cancer Institute, 2013.

40 Lund JL, Stürmer T, Harlan LC, et al: Identifying specific chemotherapeutic agents in Medicare data: a validation study. Med Care 2013; 51:e27-e34.

41 National Cancer Institute, Procedure Codes for SEER-Medicare Analyses. http://applied research.cancer.gov/seermedicare/consider ations/procedure_codes.html (accessed March $28,2014)$.

42 Searle SR, Speed FM, Milliken GA: Population marginal means in the linear model: an alternative to least squares means. Am Stat 1980;34:216-221.

43 Verzani J: Getting started with RStudio: an integrated development environment for R. Sebastopol, 2011.

44 Pisoni RL, Fuller DS, Bieber BA, Gillespie BW, Robinson BM: The DOPPS practice monitor for US dialysis care: trends through August 2011. Am J Kidney Dis 2012;60:160165.

45 Tanhehco YC, Berns JS: Red blood cell transfusion risks in patients with end-stage renal disease. Semin Dial 2012;25:539-544.

46 Eschbach JW, Abdulhadi MH, Browne JK, et al: Recombinant human erythropoietin in anemic patients with end-stage renal disease. Results of a phase III multicenter clinical trial. Ann Intern Med 1989;111:992-1000. 